\title{
Factors Affecting RFId Adoption in a Vertical Supply Chain: The Case of the Silk Industry in Italy
}

\author{
Cristina Quetti ${ }^{2}$ and Federico Pigni ${ }^{1}$ \\ ${ }^{1,2}$ Università Carlo Cattaneo - LIUC, Lab\#ID, Italy \\ cquetti@liuc.it, fpigni@liuc.it
}

\begin{abstract}
RFId adoption process is receiving a lot of attention in literature; studies assessing its potentials in supply chains are now well documented. Despite this rising interest, the diffusion pattern of RFId systems in the vertical supply chains has been only scarcely addressed and theoretical contributions explaining dynamics and drivers are still missing. This study shows that the Diffusion of Innovation Theory can be effectively used to explore these questions in a vertical SC. However, three major considerations emerged: 1) the factors influencing, facilitating or inhibiting the adoption process change over time and during the adoption process; 2) facilitators such as business associations play an important role in the supply chains composed of SMEs; 3) the position of adopters within the SC greatly influences the diffusion process.
\end{abstract}

Keywords: Interorganizational Systems, RFId, Diffusion of Innovation Theory, supply chain.

\section{Introduction}

The increasing complexity and turbulence of markets are notably shaping a fiercely competitive business environment. Besides, worldwide mobility of labor and capital are extending companies' value chains, increasing the difficulties in managing business processes [1]. Information and communication technologies (ICT) provide the needed support to enable the collaboration among trading partners to face this new competitive context. Radio Frequency Identification systems (RFId) gained attention both from researchers and practitioners, as an interesting emerging technology capable of enabling cross-company information flows integration. However, supply chainSC wide RFId system implementations are scarcely documented; the adoption process, in particular, is lacking the necessary understanding of patterns and dynamics when refereed to the inter-organizational context [2]. With this paper, we aim at filling this gap exploring the main factors driving RFId adoption across a vertical SC, assuming that these factors represent the rationale behind the diffusion of RFId systems, thus influencing and eventually predicting the intent to adopt. To answer this research question we first review past conceptual and empirical research on IOS adoption. Then we propose an explorative model, based on the Diffusion of Innovation Theory (DoI) and through qualitative methods, we seek confirmation of the validity of the main constructs through seven case studies. 


\section{Literature Review}

The literature dealing with inter-organizational system (IOS) adoption is vast. IOS adoption is an organizational-level decision that has to be considered in an interorganizational perspective, where synchronization and alignment between an organization and its trading partners become significant [3]. Most of the past studies adopted the DoI [4] to outline the attributes of an individual new technology and tools that drive its adoption[5-9]. RFId adoption studies have been built on the base of these previous findings and have only been examined recently. In comparison to former researches, they put a greater emphasis on the assessment of the interdependencies. This is particularly true as RFId systems affect information sharing at several levels (operational, tactical and strategic) and are capable of supporting different SC collaboration practices. In a recent article, [10] integrated the models proposed by [11] [9] and adapted them to explore adoption in RFId context. Another stream of research on RFId adoption focuses on the assessment of the potential benefits [12-14] and business performance of RFId applications [15], exploring the business value and drivers influencing the investment decision [16] [17-18]. Finally, other studies investigate RFId adoption on the base of specific case studies [19-21].

Despite these studies tried to frame the complexity of the inter-organizational adoption process, they focused on the assessment of the diffusion pattern on the horizontal dimension, comparing similar companies within the same industry, or framing the problem in an aggregated manner. This simple fact underlines an important emerging issue: the diffusion pattern of RFId systems in the vertical SC has been only scarcely addressed; theoretical contributions on the topic are still missing noteworthy in the understanding of its dynamics and drivers. In the following section, we present a model, structured on the DoI theory, to study the adoption drivers of RFId systems across a vertical SC.

\section{Framework}

Based on the findings of previous IOS adoption studies, three elements - external environment, organizational readiness, perceived benefits- were identified as the main drivers of adoption [3, 9-10]. In addition to these elements, we propose to include "supply chain readiness" as a new driver to account for the forces influencing SC dynamics for RFId adoption defined on the base of the studies by [22] [10] [23].

Thus the proposed model is composed of four main factors influencing RFId adoption: the external environment, the organizational readiness, the perceived benefits and SC readiness. The following section will detail in succession each factor and the hypothesis tested. In the tables we describe each factor, detailing its sub-constructs. This work is the result of a literature analysis covering all the studies published in the main IS journals and conferences on RFId adoption. Among these, we selected and proposed those factors which are supposed to be relevant drivers for adoption decision for enterprises belonging to the same SC. 


\subsection{External Environment}

External environment covers all pressures coming from the different forces shaping the business environment of the SC. Usually, political, economics, sociological and technological dynamics can affect companies' competitiveness and performances.

The external environment is composed of strategic and institutional drivers.

\section{Strategic factor}

Strategic factors encompass market dynamics and deals with those forces that in a SC stimulate a better market positioning through enhancement based on IT (in this case RFId) adoption and exploitation. We propose four main sub-constructs shaping this factor; we state the name of the sub-construct, its short description and reference.

Table 1. Strategic factors

\begin{tabular}{|l|l|l|}
\hline H1-1 & \multicolumn{1}{|c|}{$\begin{array}{c}\text { Technology } \\
\text { Maturity }\end{array}$} & {$[4,10,19,23-24]$} \\
\hline H1-2 & $\begin{array}{c}\text { Competitive } \\
\text { Pressure }\end{array}$ & {$[3,8-10,19,24-31]$} \\
\hline H1-3 & Industry & {$[10,19,25,32]$} \\
\hline H1-4 & Emulation Effect & {$[10,24,33]$} \\
\hline
\end{tabular}

\section{Institutional factors}

Enterprises can be persuaded to adopt RFId systems pressed by socio-political forces. Regulatory institutions, trade and industry associations, laws and regulations can effectively influence adoption's decisions [34]. We identified four sub-construct capable of influencing the RFId adoption process as detailed in the table below:

Table 2. Institutional factors

\begin{tabular}{|l|c|l|}
\hline H1-5 & Normative & {$[10-11,33,35]$} \\
\hline H1-6 & Incentives & {$[2,10]$} \\
\hline H1-7 & Supervisor & {$[33]$} \\
\hline H1-8 & Privacy & {$[36][27]$} \\
\hline
\end{tabular}

\subsection{Organization Readiness}

Organizational readiness refers to the internal resources that a company could commit to supporting RFId adoption. Studies on EDI outlined that financial and technological resources are the main adoption drivers [9]. However, managerial drivers were found significant in influencing the process [8] [10]A lack of internal resources even in a single company, could limit RFId potential for the entire SC.

\section{Financial resources}

Financial resources consist of the monetary resources the organization can commit to covering RFId investment and costs. 
Table 3. Financial resources

\begin{tabular}{|l|l|l|}
\hline H2-1 & Financial resources & {$[3,9-10,19,22,24,35,37]$} \\
\hline
\end{tabular}

\section{Technological factors}

Technological factors concern the implicit characteristics of the RFId system (such as complexity and compatibility), the infrastructural dimension and refer to the development and usage of the inter-organizational technology [9].

Table 4. Technological factors

\begin{tabular}{|l|l|l|}
\hline H2-2 & Complexity & {$[3-6,8-9,11,24-25,31,33]$.} \\
\hline H2-3 & Compatibility & {$[3-6,8-9,11,24-25,31,33,38]$} \\
\hline H2-4 & IS infrastructure & {$[3-6,8-9,11,24-25,31,33,38-39]$} \\
\hline
\end{tabular}

Top management support and company size

Top management support and company size are fundamental characteristic of an organization, which could favor or thwart RFId adoption. Its support is required to facilitate change during transition phases and for project sponsorship. Company size is often related to the actual availability of resources: larger companies could easily commit in comparison to smaller ones to innovative projects.

Table 5. Organizational factors

\begin{tabular}{|l|l|l|}
\hline H2-5 & Top Management & {$[3,8-10,19,25,31-35,40-43]$} \\
\hline H2-6 & Company Size & {$[8,23,31,37,44-45]$} \\
\hline
\end{tabular}

\subsection{Perceived Benefits}

The perception of the potential benefits has been traditionally considered one of the main drivers behind IOS adoption. In the scope of RFId inter-organizational adoption, perceived benefits plays a relevant role, too. In a vertical SC, benefits usually are related to business process effectiveness and efficiency improvements, which in turns could generate operational performance gains and bullwhip effect reduction.

Table 6. Perceived benefits/costs

\begin{tabular}{|l|l|l|}
\hline H3 & Perceived benefits /Costs & {$[3,7-10,12-13,15,19,25,31,35,40,46-47]$} \\
\hline
\end{tabular}

\subsection{Supply Chain Readiness}

RFId technology has a great potential in supporting interorganizational collaboration, information sharing and material flow coordination in the SC.

Moreover, adoption decisions in vertical supply chains are largely influenced by the dynamics emerging among trading partners.

Factors describing SC readiness are illustrated in the table below. 
Table 7. Supply chain readiness factors

\begin{tabular}{|l|l|l|}
\hline H4-1 & Bargaining Power & {$[3,8-10,13,19,38,46,48-52]$} \\
\hline H4-2 & Initiators & {$[33,35]$} \\
\hline H4-3 & Trust & {$[3,33,35,38,43,46,49-51,53]$} \\
\hline H4-4 & Partner ICT readiness & {$[3,24,32,35,43]$} \\
\hline H4-5 & IO Dependencies & {$[38,43,51,54]$} \\
\hline
\end{tabular}

\section{Methodology}

The aim of this work is to identify and explore the adoption drivers outlined in the model in the context of a vertical SC. The explorative case study methodology was found appropriate, investigating "a contemporary phenomenon within its real-life context, especially when the boundaries between phenomenon and context are not clearly evident", [55] a typical occurrence in studies dealing with inter-organizational issues. The study focused on a SC operating in the silk-textile Italian cluster of Como. Textile industry was identified a good candidate for the study as it shows a great potential and interest for RFId technology as it fits their needs to improve product traceability along the SC and to certify products' origin thus safeguarding the "Made in Italy" distinctive value. The analyzed SC is composed of seven SMEs each specialized in a specific process: weaving, purge, dyeing, printing, finishing and tailoring of high quality women dresses. The figure below illustrates the positioning of the companies interviewed along the Sc (names and the stage they act):

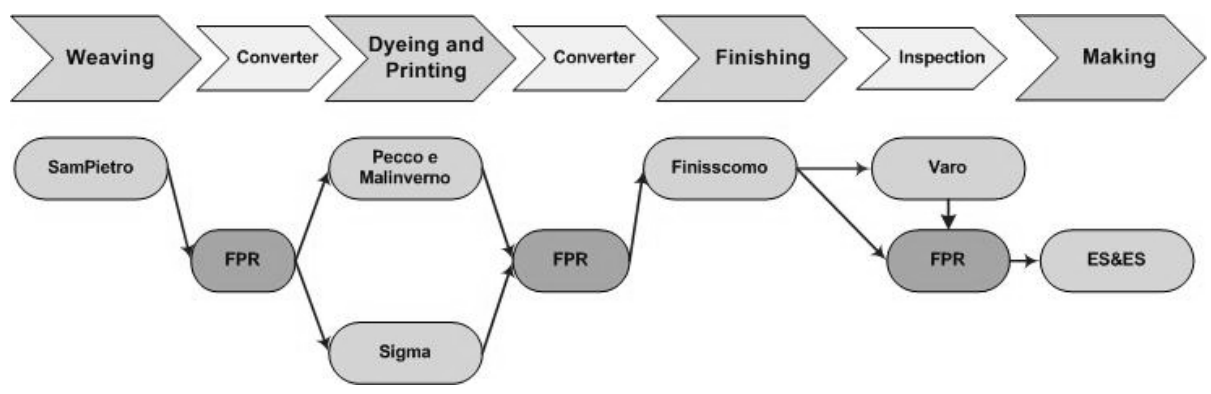

Fig. 1. The vertical supply chain

These companies performed a feasibility study and a tested in a pilot project the opportunity to implement RFId technology in their supply chain. They decided to apply RFId tags on the fabrics manufactured by Sampietro and to preserve them until the end of the chain. The information regarding the fabric are shared in real time thanks to the memory of the RFId tags and through a web service.

Data were gathered through in depth semi-structured interviews of executives and CIO across the seven organizations. Responded bias was avoided by involving multiple respondents in each organization. All direct interviews were performed between June and July 2008 and took place at companies' premises. Usually, interviews lasted 2-3 hours. The interviews were supported by a questionnaire developed on the base of 
the proposed model; they were recorded and then summarized. The interviews, then, provided the qualitative background to adopt an "interpretative" approach in this explorative study.

\section{Results}

\section{External Environment}

Concerning strategic factors and H1-1, technology maturity was not found to be a relevant inhibitor of RFId adoption. RFId vendors resulted capable of providing proper solutions to meet SC actors' needs, contrary to [10] findings.

Consistent with H1-2, our results show that competitive pressure strongly influences companies belonging to the same SC in adopting the new technology. In particular, companies confirmed that they are aiming at creating a sustainable competitive advantage leveraging RFId traceability potential. FPR owner: "competitive environment forces us towards the research of new technologies capable of supporting item traceability, recording weaves history along the entire SC and demonstrating the Italian origin of the product. That's why we decided to adopt RFId systems"; Pecco e Malinverno TM:"in this downturn time, we are seeking innovations to enhance company's agility and counter Asian competitors; A SC wide RFId implementation fits our needs". According to Henderson and Vankatramam [56], RFId is a tool for strategy execution influencing internal IS infrastructure and process (internal), in order to fit the objective of ensuring fabrics origin and enhance SC relationship for a effective collaborative activity planning and a better customer transparency.

H1-3 was confirmed, too. We found that the presence of existing RFId "closed loop" implementation within the industry, actually positively influenced the propensity for adoption of other actors within the SC (existing experiences demonstrated the ability of tags to survive to several aggressive industrial processes as the printing, purge or pressing). On the contrary, $\mathrm{H} 1-4$, investigating contagion, was found not applicable as SC wide implementations within the industry were still missing.

The Normative institutional element, H1-5, despite significant in other contexts, resulted poorly relevant as Italian regulation leaves to manufacturers the choice to voluntary adhere or propose a traceability system.

Interestingly, confirming H1-6, revealed that the presence of a "supervisor" facilitate, and in several situations actually permitted, cross-company adoption. The project champion from UNICO (Confindustria Como, the local business association) responsible for overseeing the adoption process, guaranteeing and controlling the fairness of the implementation was decisive. The Top Management of Pecco and Malinverno commented: "supervisor persuaded our participation in the project and his presence confirmed its importance".

Moreover, the involvement of a University Lab in the project effectively supported RFId knowledge transfer and eased the process: university researchers led the feasibility study and took care of the supporting activities towards SC participants.

Incentives favored RFId inter-organizational adoption confirming H1-7. The public incentives provided by Lombardy Region convinced hesitating partners by covering half of investment costs: companies were at the same time reducing their expenses and "reassured" of the political relevance of the innovation effort. Contrary to common 
understanding, but consistent with studies on RFId implementations supporting B2B transaction, H1-8 was not confirmed. Privacy was considered relevant only for those goods impacting directly the consumer.

\section{Organizational readiness}

The weight of Financial resources in influencing RFId adoption were found less relevant to adoption than expected. We are not supporting that the financial capability of companies to commit to the investment is irrelevant, but it did not impact the SC favoring or inhibiting adoption. This result confirms [39] study in which they demonstrated that RFId adoption is not strictly determined by the sheer amount of financial resources, but by the investment expectations. Actually we found that ROI can show only a window of investment returns: other index, such as KPI, can measure an early return on the RFId investment. Respondents generally confirmed that RFId complexity was low (H2-2) requiring only incremental process improvements.

H2-3, compatibility, was found capable of affecting RFId adoption. Those companies that already had an identification system in place (such as a barcode one) showed less compatibility problems in integrating and adapting their systems with those of other companies. Scarce IS infrastructure was found to be a inhibitor factor for RFId adoption, confirming H2-4. Sigma TM: "our company has not the expertise to support cross-company RFId implementation".

TM support (H2-5) was indicated as fundamental by all the companies interviewed and in several occasion suggested to be critical for achieving project goals. Firm size, H2-6, was effectively influencing RFId adoption.

\section{Cost/Benefits}

The distribution of cost and the appropriability of the generated benefits were found significant for the adoption process along the analyzed SC (H3). Benefits, as much as costs, were not evenly distributed among participants, as already assessed in previous studies [13]. In particular, we found that companies at the edge of the downstream of the supply chain experienced more advantages related to the information sharing enabled by the RFId system, than the other companies; implementation expenditures resulted similar along the SC and largely dependent on the existing IS infrastructure.

\section{SC Readiness}

Bargaining power of partners, $\mathrm{H} 4-1$, resulted decisive in persuading companies to participate to the system and was mainly exerted through recommendations [9].

Trust among trading partners, ( $\mathrm{H} \mathrm{4-2)}$ built on top of a solid relationship and a recognized competence, was effectively contributing to the success of the adoption process; Pecco and Malinverno TM commented: "FPR owner visit our plants three times per week. We trust him and we thing that tools like RFId technology, capable of enabling information sharing, would help our collaboration".

The presence of an initiator, was confirmed to favor adoption (H 4-3). FPR owner, whose company is positioned at the center of the SC, after perceiving the business opportunity for an RFId based SC traceability system, actively involved other companies in the project. Its first effort, he told us, was frustrated by partners' low ICT readiness, thus confirming that in a SC the companies with low ICT can slow down both the process and hinder the benefits emerging from the project ( $\mathrm{H} \mathrm{4-4).}$ 
Surprisingly, H 4-5, SC dependencies were found less significant than other factors in favoring RFId adoption. In particular, in the analyzed SC companies have stable relationship, factors like the bargaining power of the partner, or the trust among them resulted to drive more the adoption process than more institutionalized contracts.

\section{Conclusions and Discussion}

Consistently with our expectation, we found that external environment, organization readiness, cost/benefits sharing and SC readiness are the all driving factors of RFId adoption even in a vertical SC. However, in this context only of the several factors identified were found significant.

Our findings suggest that competitive pressure strongly influence managers' adoption decision [19]. The chance to improve competitiveness through RFId items traceability drove the initiator (FPR) to assess the feasibility of RFId, involving other companies in the SC. However, companies presenting low technological compatibility and inadequate IS infrastructures nearly stopped the project (Sigma and Finisscomo); companies were after encouraged, mainly from the incentive, to align their technological level to that of their partners [3-4, 9-10, 19].

Other decision elements, underlying a life cycle for the decision making process of the RFId adoption, appeared later in the project: those companies showing good organizational readiness based their decision on the evaluation of cost/benefits (Sampietro, FPR, Pecco e Malinverno, Varo and ES\&ES). This assessment discouraged the company located on the upstream of the supply chain (Sampietro), which decided, in a first time, to give up the project as implementation costs were not balanced by corresponding benefits (they were supposed to tag items) [13]. Only agreeing on a "fair" division of costs, supported by a compensation of tag costs and application, had the project running. This suggest that the position of a company within the supply chain is a strong determinant of adoption, thus partially hindering DoI application in this context. The presences of a supervisor and the availability of financial incentives were confirmed as strong facilitator of adoption [33]. Without a supervisor, not only the implementation would have failed, but also some of the partners would not have committed the resources for the feasibility study. Moreover, the supervisor - the business association - acted as a guarantor of the stakes of all the parties involved and of adoption fairness. The bargaining power of certain actors was actively used, suggesting it as a critical factor of adoption. FPR recommended [9] Sigma and Finisscomo to participate to RFId, although these companies were reluctant. The existing trust between FPR and Pecco e Malinverno and based on competences [49] enabled the cross-company adoption, as both companies saw the RFId project as a chance to enhance collaboration.

In conclusion, this study effectively supports the use of the DoI Theory to study the diffusion of RFId in a the specific context of vertical supply chains, an aspect still unexplored in literature. However, several observation should be noted. Firstly, despite the majority of factors were found significant, their relevance changed in time. The more the project progressed, the factors influencing, facilitating or inhibiting the 
adoption process changed their importance and companies changed their mind as soon as actions were taken to correct the emerging deadlocks. Moreover, even when all members of the SC decide to adopt the RFId system, and that performance gains have been demonstrated the real commitment of companies and their TM is needed to enact collaboration and information sharing.

\section{References}

1. Friedman, T.L.: The World is flat: A Brief History of the Twenty-first Century. Farrar, Straus and Giroux, New York (2005)

2. Kauffman, R.J., Curtin, J., Riggins, F.J.: Making the most out of RFId technology: a research agenda for the study of the adoption, usage and impact of RFId. Management Science 40(8) (2005)

3. Chwelos, P., Benbasat, I., Dexter, A.S.: Empirical Test of an EDI Adoption Model. Information System Research 13(3), 304-321 (2001)

4. Rogers, E.M.: Diffusion of innovations, 5th edn. Free Press, New York (2003)

5. Cooper, R.B., Zmud, R.W.: Information technology Implementation Research: a Technological Diffusion Approach. Mgt. Sc. 36(2), 123-139 (1990)

6. Moore, G.C., Benbasat, I.: Development of an instrument to measure the perceptions of adopting an information technology innovation. Information Systems Research 2(3), 192 $222(1991)$

7. O'Callaghan, Kaufmann, Konsynski: Adoption correlates and share effects of electronic data interchange systems in marketing channels. Journal of Marketing 4, 45-56 (1992)

8. Premkumar, G., Ramamurthy, K.: The role of Interorganizational and Organizational factors on decision mode of adoption of Interorganizational Systems. Decision Science 26, 75-86 (1995)

9. Iacovou, C.L., Benbasat, I., Dexter, A.S.: Electronic data interchange and small organizations: Adoption and impact of technology. MIS Quarterly 19(4), 465-485 (1995)

10. Sharma, A., Citurs, A., Konsynski, B.: Strategic and Institutional Perspectives in the Adoption and Early Integration of Radio Frequency Identification (RFID). In: Proceeding the 40th Hawaii International Conference on System Science, Hawaii, USA. IEEE, Los Alamitos (2007)

11. Teo, H.H., Wei, K.K., Benbasat, I.: Predicting Intension to Adopt Interorganizational Linkages: An Institutional Perspective. MIS Quarterly 27(1) (2003)

12. Baars, H., et al.: Profiling benefits of RFId applications. In: Americas Conference on Information System, AMCIS 2008 (2008)

13. Bensel, P., et al.: Cost-Benefit in cross-company RFId application: a case study approach. In: International conference of Information System, Paris, France (2008)

14. Pigni, F., Ugazio, E.: Measuring RFId Benefits in Supply Chains. In: Proceedings of the Fifteenth Americas Conference on Information Systems, San Francisco, California (2009)

15. Gille, D., Strüker, J.: Into the Unknown: Measuring the Business Performance of RFID Applications. In: Proceedings of the 16th European Conference on Information Systems, Galway, Ireland (2008)

16. Becker, J., et al.: Calculating the Process Driven Business Value of RFID Investments - A Causal Model for the Measurement of RFID Technologies in Supply Chain Logistics. In: Proceedings of the Fourteenth Americas Conference on Information Systems, Toronto, ON, Canada (2008) 
17. Ivantysynova, L., Ziekow, H., Gunther, O.: A guide to Asses Cost and Benefits for RFId Investments in Manufacturing. In: Proceeding of ICIS 2007 Workshop on E-business, Montreal, Canada (2007)

18. Tellkamp, C.: The impact of Auto-ID technology on process performance - RFID in the FMCG supply chain. Dissertation of University of St.Gallen, Graduate school of business Administration (2006)

19. Krasnova, H., Wezer, L., Ivantysynova, L.: Adoption in the Automotive Industry. In: Americas Conference of Information System, AMCIS (2008)

20. Tewary, A., Kosalge, P., Motwani, J.: Piloting RFID in an Aerospace and Defense Supply Chain. In: Proceedings of the Fourteenth Americas Conference on Information Systems, Toronto, ON, Canada (2008)

21. Loebbecke, C., Huyskens, C.: Towards Standardizing Success: RFID in Fashion Retailing. In: BLED Proceedings, Bled, Slovenia (2006)

22. Ranganathan, C., Jha, J.: Adoption of RFID Technology: An Exploratory Examination from Supplier's Perspective. In: Proceedings of the Eleventh Americas Conference on Information Systems, Maha, NE, USA (2005)

23. Madlberger, M.A.: A Model of Antecedents of RFID Adoption Intention in the Supply Chain. In: Proceedings of the 42nd Hawaii International Conference on System Sciences (2009)

24. Fichman, R.G.: Going beyond the dominant paradigm for information technology innovation research: Emerging concepts and methods. Journal of the Association for Information Systems 5(8), 314-355 (2004)

25. Brown, I., Russel, J.: Radio Frequency Identification Technology: An exploratory Study on Adoption in the South African Retail Sector. International Journal of Information Management 27(4), 250-265 (2007)

26. Chau, P.Y.K., Tam, K.Y.: Factors Affecting the Adoption of Open Systems: An Exploratory Study. MIS Quarterly 21(1), 1-24 (1997)

27. Chin, W.W., Johnson, N., Schwarz, A.: A fast Approach to Measuring Technology Acceptance and Other Constructs. MIS Quarterly 32(4), 687-703 (2008)

28. Davis, F.D.: Perceived Usefulness, Perceived Ease of Use, and User Acceptance of Information Technology. MIS Quarterly 13(3), 319-340 (1989)

29. Mithas, et al.: How Strategic Posture and Competitive Environment Influence Firms' IT Investments: Theory and Evidence. In: Proceedings of the 30th International Conference on Information Systems (2009)

30. Porter, M.E., Millar, V.E.: How information gives you competitive advantage. Harvard Business Review (1985)

31. Seymour, L., Porter, E.L., Willuweit, L.: RFID Adoption into the Container Supply Chain: Proposing a framework. In: Proceedings of the 6th Annual ISOnEworld Conference, Las Vegas, NV (2007)

32. Morrel, M., Ezingeard, J.N.: Revisiting adoption factors of inter-organizational information systems in SMEs. Logistics Information Management 15(1), 46-57 (2002)

33. Karahanna, E., Straub, D.W., Chervany, N.L.: Information technology adoption across time: A cross-sectional comparison of pre-adoption and post-adoption beliefs. MIS Quarterly 23(2), 183-213 (1999)

34. Goswami, S., Teo, H.H., Chan, C.: Decision-Maker Mindfulness in IT Adoption: The Role of Informed Culture and Individual Personality. In: AMCIS (2009)

35. Robey, D., Im, G., Jonathan, D.W.: Theoretical foundations of empirical research on interorganizational systems: assessing past contributions and guiding future directions. Journal of the Association for Information Systems 9(9), 497-518 (2008) 
36. Brown, M., Patadua, S., Dua, S., Meyers, M.: Comptia RFId plus passport. McGraw-Hill, New York (2007)

37. Lee, J.H., Kim, C.O.: Multi-agent systems applications in manufacturing systems and supply chain management: a review paper. International Journal of Production Research 46(1), 233-265 (2008)

38. Saunders, C., Hart, S.: Elettronic Data Interchange Across Organizational Boundaries: Building a theory of motivation and implementation. In: Administrative Sciences Association of Canada Twenty First Annual Conference, Lake Luise, Alberta, Canada (1992)

39. Whitaker, J., Mithas, S., Krishnan, M.S.: A Field Study of RFID Deployment and Return Expectations. Production and Operations Management 16(5), 599-612 (2007)

40. Byrnes, J.L.S., Shapiro, R.D.: Intercompany operating ties: unlocking the Value In Channel Restructuring. Sloan Management Review 32, 15-18 (1991)

41. Klonglan, G.E., Coward, E.W.: The Concept of Symbolic Adoption: A Suggested Interpretation. Rural Sociology 35(1), 77-83 (1970)

42. Kunnathur, A.S., Vaithianathan, S.: RFID Adoption: An Exploratory Study of Issues and Concerns. In: 2008 AMCIS Proceeding (2008)

43. Mentzer, J.T.: Fundamentals of Supply Chain Management. Sage Publications, Inc., Thousand Oaks (2004)

44. Patterson, K.A., Grimm, C.M., Corsi, T.M.: Adopting new technologies for Supply Chain. Logistics and Transportation Review 39(2), 95-121 (2003)

45. Crum, M.R., Premkumar, G., Ramamurthy, K.: An assessment of motor carrier adoption, use, and satisfaction with EDI. Transportation Journal 35(4), 44-57 (1996)

46. Madlberger, M., Roztocki, N.: Digital Cross-Organizational Collaboration: Towards a Preliminary Framework. In: Proceeding of the Fifteenth Americas Conference on Information Systems, San Francisco, CA (2009)

47. Pfeiffer: The Diffusion of Electronic Data Interchange. Springer, N.Y. (1992)

48. Carter, J.R., Slaight, T.H., Blascovich, J.: Technology collaboration, supply chain design. Supply Chain Management Review (2007)

49. Ghosh, A., Fedorowicz, J.: Governance Mechanisms for Coordination and Information Sharing in Supply Chains: The Role of Trust. In: AMCIS Proceeding (2005)

50. Hart, P., Saunders, C.S.: Power and Trust: Critical factors in the adoption and use of electronic data interchange. Organizational Science 8(1), 23-42 (1997)

51. Klein, R., Rai, A.: Interfirm strategic information flows in logistic supply chain relationship. MIS Quarterly 33(4), 735-762 (2009)

52. Son, J., et al.: Understanding the Development of IOS-Based Trading Partner Relationships: A Structural Model and its Empirical Validation. Journal of Organizational Computing and Electronic Commerce 18(1), 34-60 (2008)

53. Rai, A., et al.: Firm performance impacts of digitally enabled supply chain integration capabilities. Mis Quarterly 30(2), 225-246 (2006)

54. Schaefermeyer, M., Rosenkranz, C.: Inhibiting Factors for Adopting Enterprise Systems in Networks of Small and Medium-Sized Enterprises - An Exploratory Case Study. In: AMCIS (2008)

55. Yin, R.K.: Case Study Research. In: Design and Methods, 3rd edn. Sage Publications, California (2003)

56. Henderson, J.C., Vankatramam, N.: Strategic Alignment: leveraging Information Technologies for transforming organizations. IBM System Journal 32(1), 472-484 (1993) 